\title{
Laser-induced ultrasonic waveform derivation and transition from a point to a homogeneous illumination of a plate
}

\author{
Jernej Laloš*, Matija Jezeršek, Rok Petkovšek, Tomaž Požar \\ University of Ljubljana, Faculty of Mechanical Engineering, Aškerčeva 6, 1000 Ljubljana, Slovenia
}

\begin{abstract}
Ultrasound modeling, being an established practice, is used to study the fundamentals of light-matter interactions. Although much has been published on the matter, pressure and thermal expansion induction mechanisms in laser ultrasonics have rarely been combined, as they should, in a single ultrasonic source while the effects of its size variation have only been shown to a limited extent. In the paper, we unite these light-matter interaction mechanisms, with inclusion of lateral optical forces, into a single laser-stimulated source as it is observed in nature. With a laser pulse as a manipulable source, we simulate the multifaceted workings of light-matter interactions by exposing the distinct transients originating from different source localities as generated by different induction mechanisms. We also present a transition of simulated ultrasonic waveforms in the epicentral point on the surface of a solid plate opposite from the source while it is expanded from a point to a quasi-limitless extent for pressure and thermal expansion generation regimes. The model utilizes geometric probability theory together with Huygens' superposition principle and temporal convolutions to construct the desired waveforms out of individual Green's functions. We show how the ultrasound generation regimes stem out of a single source and how its size together with energy and momentum transfers during the light-matter interactions affect the induced ultrasonic transients.
\end{abstract}

Keywords: laser ultrasonics, optical forces, radiation pressure, thermoelastic expansion, waveform modeling

\section{Introduction}

With thermo-elastodynamic laws generally well understood, with advanced mathematical tools and powerful computing machines, modeling of wave propagation has become an established practice. In ultrasonic research and associated applications, it is essential for interpreting measurements and understanding the underlying physical mechanisms. Due to recent strides in fundamental research, particularly regarding the measuring of light-pressure-induced ultrasound $[1,2]$ and the so-called Abraham-Minkowski controversy $[3,4,5,6,7,8,9,10,11]$, laser ultrasound modeling is gaining a new momentum and a novel aspect in its applicability.

The use of a laser pulse to stimulate an ultrasonic source in elastic media provides the ability to easily control its time profile, spatial distribution, energy, and momentum in much broader ranges than using mechanical

\footnotetext{
* Corresponding author.

Email address: jernej.lalos@fs. uni-lj.si (Jernej Laloš)
}

ultrasonic sources. An additional and unique aspect of laser ultrasonics is that it provides one of the platforms from which to study the intricacies of light-matter interactions.

While some condensed volumes on the matter in general have been compiled [12, 13, 14, 15, 16, 17, 18], most of the relevant research is mainly scattered in time and throughout literature. One can find, for example, expositions on pressure-induced [19, 20, 21, 22, 23, 24, $25,26,27,28,29,30,31,32,33,34,35]$ as well as thermal-expansion-induced $[26,27,28,29,30,31,32$, $33,35,36,37,38,39,40,41,42,43,44]$ ultrasound propagation in a half-space $[21,22,23,24,25,26,27$, $28,32,37,42,43]$ or in a large plate $[19,20,29,30$, $31,32,33,34,35,36,37,38,39,40,41,44]$ from point [26, 27, 28, 29, 30, 31, 32, 33, 34, 37, 38, 39], areal $[20,21,22,23,24,25,35,36,40,41,42]$ and quasi-limitless [19, 43, 44] sources to receivers in similar ranges in lateral [19, 37, 42], epicentral [32, 33, 34, 35, 36, 37, 38, 39, 43, 44] or arbitrary $[20,21,22,23,24,25,26,27,28,29,30,31,40,41]$ relative positions. 
In this, to the best of our knowledge, there is a noticeable gap in seamlessly connecting the range of sources into one smooth transition from a point-source limit, through the areal-source formation, to the limitlesssource (one-dimensional) limit. Combing through the forementioned literature reveals that each of these produces specifically shaped transient waveforms which are so dissimilar in their appearance that this kind of limit-to-limit transition becomes unintuitive while a comparison of pressure and thermal expansion sources reveals a fundamental generation mechanism difference.

In a large plate, for example, it can be observed that the epicentral waveforms in a point-source limit consist of variety of peaks, different for pressureinduced and thermal-expansion-induced regimes. Towards the one-dimensional limit, however, the waveforms in the pressure-induced regime appear to consist of steps and the waveforms in the thermal-expansioninduced regime remain flat with only small precursor peaks. From this it can be concluded that, in a pointsource limit, the waveforms are composed of all incoming wave types while, in the one-dimensional limit, only one type of P-waves causes the disturbances. More interestingly, the step-like waveforms in the pressureinduced regime indicate that linear momentum has been transferred to the plate, whereas the waveforms in the thermal-expansion-induced regime indicate no such occurrence.

Demonstrating such a seamless limit-to-limit transition in source dimensions and its effects on the ultrasonic waveforms, at least in theory, would not only complete a gap in knowledge but would also garner a new understanding of the nature of ultrasonic waves. In particular, it would expose the transients originating from different source localities and their superposition in the inter-limit arrangement.

A laser-stimulated source, in general, combines light pressure, thermal expansion due to light absorption, electrostriction, and material ablation as means of producing ultrasonic waves. Each of these mechanisms may be experimentally fairly well isolated with appropriate selection of elastic materials and their surface treatments, laser energies and wavelengths $[1,2]$.

Since pressure and thermal expansion generation regimes have been often treated separately, their mathematical formulation from a common origin has rarely been consistently derived. More than curiosity, such a common derivation would show a physical connection between the regimes and demonstrate the true multifaceted workings of light-matter interactions in nature.

Drawing additional inspiration from the analogies with seismology $[45,46,47,48,49,50]$, we derive a formulation that combines the light-pressure and lightabsorption effects in a laser-illuminated volume on a solid surface and treats it as common ultrasonic source. We show how such a source, through a series of transfer functions, is responsible for material displacement in elastic material. The presented formulation [51, 52, 53] utilizes Huygens' superposition principle that is statistically stremlined by means of geometric probability theory [54] for constructing the required volumeto-volume transfer functions out of weighted and superimposed point-to-point Greens' functions. The desired waveforms are then obtained from them by temporal convolutions with the source inputs. In our case, the Green's functions were originally derived by Hsu [31]. This general formulation is as a simple method for obtaining sufficiently detailed volume-to-volume transfer functions and subsequent displacement waveforms while accounting for arbitrary source and observation distributions [51, 52, 53].

We also present simulations of successive ultrasonic waveforms (time evolutions) in a plate originating from a surface source which is enlarged from a single point to a quasi-limitless extent (homogeneous illumination). Both, pressure (elastic) and thermal expansion (thermoelastic) induction mechanisms are mathematically derived and simulated as if they were stimulated by a uniformly distributed laser pulse acting upon a circular area on the surface of a glass plate with the observation point set in the epicentral position on the opposite plate surface.

The results are visualized as three-dimensional line plots and shaded surface plots as well as animated videos available as supplementary material on-line. Particular attention is given to individual wave arrivals and their effects on the waveforms. Selected simulated waveforms are compared to the independent models from the literature $[2,20,40]$.

In addition to being a theoretical study of ultrasound generation and propagation, the purpose of such simulated waveforms is to introduce well formulated underlying physical concepts and mechanisms to the interpretation and evaluation of the corresponding experimental data. In a particular experimental setup, the measured sensor signal should be compared to the simulated waveforms modeled for the same geometric arrangement, the same materials, and the same stimulating laser pulse, while accounting for the sensor transfer function as well. A good match between them should indicate that the correct physical assumptions have been made. Such a process was used and is described in detail in some of our previous work $[51,52]$.

Due to conceptual and theoretical nature of this paper, 
no measurements are presented. Some may be found throughout the literature $[1,2,6,12,13,14,15,16,18$, $20,24,26,30,32,33,34,35,36,39,40,42,44,51,52]$.

\section{Formulation of laser-induced ultrasound}

A consistent mathematical description of ultrasound generation mechanisms in laser ultrasonics is achieved by implementation of analogous formulations used for mechanical excitations of matter that are rooted in Green's function formalism as well as known theoretical descriptions of excitations of illuminated matter $[1,2,3,4,5,6,7,8,9,10,11,12,13,14,15,16,17,18$, $19,20,21,22,23,24,25,26,27,28,29,30,31,32,33$, $34,35,36,37,38,39,40,41,42,43,44]$. Their additional expansion from a point-to-point approximation to an arbitrary volume-to-point and volume-to-volume formulation is achieved by employing statistically streamlined Huygens' and superposition principles by means of geometric probability theory $[51,52,53]$.

In this formulation, elementary terms are supposed to be algebraically linear in nature allowing them to be combined, like building blocks, through linear superpositions and temporal convolutions. Sources, wave propagation functions and material displacements are treated with respect to their temporal $t$, directional $\hat{\boldsymbol{x}}, \hat{\boldsymbol{y}}, \hat{\boldsymbol{z}}$, and either spatial $\boldsymbol{v}, \boldsymbol{w}=(x, y, z)$ or areal $\tilde{\boldsymbol{v}}, \tilde{\boldsymbol{w}}=(x, y)$ dependencies. Temporal convolution of two functions is represented by an asterisk symbol between them: $a(t) * b(t) \equiv \int_{-\infty}^{\infty} a\left(t^{\prime}\right) b\left(t-t^{\prime}\right) \mathrm{d} t^{\prime}$, as per common engineering convention.

\subsection{Laser-stimulated ultrasonic source}

A laser pulse is a burst of energy $E$ distributed in time $t$ over an area $\tilde{\boldsymbol{v}}$ traveling in a certain direction $\hat{z}$ with the speed of light and has an intensity of:

$$
\boldsymbol{I}(\tilde{\boldsymbol{v}}, t)=E \tilde{\eta}(\tilde{\boldsymbol{v}}) \theta(t) \hat{z} .
$$

Its areal distribution $\tilde{\eta}(\tilde{\boldsymbol{v}})$ and time profile $\theta(t)$ are normalized and regarded as separable. When it is incident on a material surface, light-matter interaction occurs.

Most prominent and commonly observable lightmatter interaction phenomena in sub-ablation regimes manifest themselves in the form of radiation pressure and thermal expansion due to light absorption. Radiation pressure exerts on a nontransparent illuminated material a momentum-transferring force in the direction normal to the incident surface (normal optical force). Conversely, thermal expansion employs omnidirectional material enlargement of a light-absorbing illuminated volume to cause force dipoles in the affected material-without net momentum transfer. Additionally, lateral optical forces (akin to electrostriction in dielectrics and magnetostriction in ferromagnetics) may be born without net momentum transfer $[3,4,7,8,9,10,11]$. These are rather more subtle, however, and are going to remain largely beyond the scope of simulations in this paper.

Subject to the nature of light-matter interaction and its distribution, a laser-illuminated area on the material surface and light-penetrated volume beneath it can, in principle, be regarded as a collection of directed force $f_{\mathrm{j}}(\boldsymbol{v}, t)$ and force dipole $m_{\mathrm{jk}}(\boldsymbol{v}, t)$ distributions which act together as ultrasound-generating source:

$$
\text { source }\left\{\begin{array}{l}
\sum_{\mathrm{j}} f_{\mathrm{j}}(\boldsymbol{v}, t) \hat{\boldsymbol{j}}, \\
\sum_{\mathrm{j}, \mathrm{k}} m_{\mathrm{jk}}(\boldsymbol{v}, t) \hat{\boldsymbol{\jmath}},
\end{array}\right.
$$

with $\mathrm{j}, \mathrm{k}=\hat{\boldsymbol{x}}, \hat{\boldsymbol{y}}, \hat{\boldsymbol{z}}$. With all of their dimensional distributions in mind, each of these can be further decomposed into: $f_{\mathrm{j}}(\boldsymbol{v}, t)=J_{\mathrm{j}} \eta_{\mathrm{j}}(\boldsymbol{v}) \theta(t)$, and $m_{\mathrm{jk}}(\boldsymbol{v}, t)=$ $D_{\mathrm{jk}} \eta_{\mathrm{jk}}(v) \Theta(t)$. Their time profiles $\theta(t), \Theta(t)$ and spatial distributions $\eta_{\mathrm{j}}(v), \eta_{\mathrm{jk}}(v)$ are considered separable and are normalized, while $J_{\mathrm{j}}$ and $D_{\mathrm{jk}}$ represent directional force impulses and strength of the force dipoles, respectively. With this, the general form of Eq. (2) is expanded to:

$$
\text { source }\left\{\begin{array}{l}
\sum_{\mathrm{j}} J_{\mathrm{j}} \eta_{\mathrm{j}}(\boldsymbol{v}) \theta(t) \hat{\boldsymbol{j}}, \\
\sum_{\mathrm{j}, \mathrm{k}} D_{\mathrm{jk}} \eta_{\mathrm{jk}}(\boldsymbol{v}) \Theta(t) \hat{\boldsymbol{j}} .
\end{array}\right.
$$

\subsection{Light-matter interaction}

Given the usual particularities of interactions between a laser pulse and elastic dielectric material, a few simplifying assumptions may be made from which the source formulation in Eq. (3) can be particularized and simplified.

Since the momentum-transferring impulse acts mostly in the direction normal to the incident surface, which is set in the $\hat{\boldsymbol{x}} \hat{\boldsymbol{y}}$-plane, its directional components can be limited to one: $\mathrm{j}=\hat{z}$. This exchange occurs on the surface from which the light is reflected and in the volume in which the light is absorbed. Reflectivity $R$, absorptivity $A$ and transmissivity $T$ of the laser light in the illuminated material are connected by: $R+A+T=1$. The entire momentum-transferring force impulse is thus collected as: $J_{\mathrm{z}}=(1-T+R) E / c_{0}$, where $E$ represents the laser pulse energy, and $c_{0}$ the speed of light in vacuum.

If, in the light-absorbing volume, material expansion is isotropic, shear dipoles are zero while tensile dipoles remain non-zero and are equal. In this case, 
the number of force dipoles is reduced to three: $\mathrm{jk}=$ $\mathrm{jj}=\hat{\boldsymbol{x}} \hat{\boldsymbol{x}}, \hat{\boldsymbol{y}} \hat{\boldsymbol{y}}, \hat{z} \hat{z}$, while their strengths of force dipoles: $D_{\mathrm{xx}}=D_{\mathrm{yy}}=D_{\mathrm{zz}} \equiv D=\gamma A E$, and their spatial distributions: $\eta_{\mathrm{xx}}(\boldsymbol{v})=\eta_{\mathrm{yy}}(\boldsymbol{v})=\eta_{\mathrm{zz}}(\boldsymbol{v}) \equiv \eta_{\mathrm{jj}}(\boldsymbol{v})$, are equalized $[13,14,35,36,37]$. Here, $A E$ represents the absorbed laser-pulse energy, with $\gamma=\alpha Y /((1-2 \mu) \rho C)$ as the Grüneisen parameter consisting of material properties of the absorbing material which are explained further in the text.

In materials, in which heat accumulation and ultrasound reverberation happen in time frames much shorter than subsequent thermal conduction and radiation, the temperature field in matter, after the light-matter interaction is finished and for the duration of observed ultrasound propagation, can be thought of as stationary. With this, the time profile of the laser pulse $\theta(t)$ can be translated directly to the source in the momentumtransferring regime, but it has to be convolved with a unit step function $H(t)$ in the energy-absorbing regime as it represents the heat accumulated over the pulse duration: $\Theta(t)=\int_{0}^{t} \theta\left(t^{\prime}\right) \mathrm{d} t^{\prime}=\theta(t) * H(t)$ [55].

Each of the spatial distributions of the source components can be further split into two independent parts: $\eta_{\mathrm{z}}(\boldsymbol{v})=\tilde{\eta}(\tilde{\boldsymbol{v}}) \zeta(z)$ and $\eta_{\mathrm{jj}}(\boldsymbol{v})=\tilde{\eta}(\tilde{\boldsymbol{v}}) Z(z)$. Their first parts are directly correlated with the areal distribution $\tilde{\eta}(\tilde{\boldsymbol{v}})$ of the incident laser pulse while the other parts represent linear depth distributions of the source in the direction of pulse propagation. The second parts are, therefore, subject to the mode of light-matter interaction as they describe the momentum-interacting layers in the momentum-transferring regime $\zeta(z)$ and the lightabsorbing layers in the energy-absorbing regime $Z(z)$.

Finally, considering all of these approximations, the laser-illuminated volume after such light-matter interaction becomes a much more specific ultrasonic source:

$$
\text { source }\left\{\begin{array}{l}
J_{\mathrm{Z}} \tilde{\eta}(\tilde{\boldsymbol{v}}) \zeta(z) \theta(t) \hat{z}, \\
D \tilde{\eta}(\tilde{\boldsymbol{v}}) Z(z) \theta(t) * H(t)(\hat{\boldsymbol{x}}+\hat{\boldsymbol{y}}+\hat{z}) .
\end{array}\right.
$$

Each of the two different generation mechanisms that constitute a single source is conceptually presented in Fig. 1 as an individual source type.

\subsection{Wave propagation}

At the core of this approach, the ultrasonic wave propagation is assumed to be well described by a set of thermo-elastodynamic equations with appropriate initial and boundary conditions which account for all relevant wave propagation effects such as attenuation, geometric spreading and scattering. These are solved, either analytically or numerically, in the confines of the Green's function formalism which yields time-resolved $t$ solutions in the form of Green's functions $g_{\mathrm{ij}}\left(\boldsymbol{v}_{0}, \boldsymbol{w}_{0}, t\right)$ or $g_{\mathrm{ij}, \mathrm{k}}\left(\boldsymbol{v}_{0}, \boldsymbol{w}_{0}, t\right)$. These are point-to-point transfer functions that transform a $\delta$-impulse input signal in the direction $\mathrm{j}$ at a point $\boldsymbol{v}_{0}$ (such as force $F_{\mathrm{j}}\left(\boldsymbol{v}_{0}, t\right)$ or force dipole $\left.M_{\mathrm{jk}}\left(\boldsymbol{v}_{0}, t\right)\right)$ to an output signal in the direction $\mathrm{i}$ at a point $\boldsymbol{w}_{0}$ (such as displacement $u_{\mathrm{i}}\left(\boldsymbol{w}_{0}, t\right)$ ). Usually, they are arranged in a matrix or a tensor. In general, Green's functions are dependent on the thermoelastic properties of the wave-carrying medium: its mass density $\rho$, Young's modulus $Y$, Poisson's ratio $\mu$, specific heat $C$, linear thermal expansion coefficient $\alpha$, and thickness $h_{0}$, as well as relative positions of the input and output points $\boldsymbol{w}_{0}-\boldsymbol{v}_{0}$. Most of these properties are considered to be constant during the wave propagation and are omitted in the common notation of Green's functions. Individual Green's functions may also be measured in a limited frequency band. Either calculated or measured, any would, in principle, suffice here.

Different kinds of input signals require different types of Green's functions to produce their displacement waveforms. The input forces $F_{\mathrm{j}}\left(\boldsymbol{v}_{0}, t\right)$ require doubleindexed Green's functions $g_{\mathrm{ij}}\left(\boldsymbol{v}_{0}, \boldsymbol{w}_{0}, t\right)$ while the input force dipoles $M_{\mathrm{jk}}\left(\boldsymbol{v}_{0}, t\right)$ require triple-indexed Green's functions $g_{\mathrm{ij}, \mathrm{k}}\left(\boldsymbol{v}_{0}, \boldsymbol{w}_{0}, t\right) \equiv \partial g_{\mathrm{ij}}\left(\boldsymbol{v}_{0}, \boldsymbol{w}_{0}, t\right) / \partial k$. In principle, higher order Green's functions $g_{\mathrm{ij}, \mathrm{kl} . . .}\left(\boldsymbol{v}_{0}, \boldsymbol{w}_{0}, t\right)$ are needed for higher order input momenta $M_{\mathrm{jkl} . . .}\left(v_{0}, t\right)$, but this is beyond the physical scope of the presented model.

When many such point-to-point Green's functions are connecting individual input points from a volume $v$ with output points in a volume $\boldsymbol{w}$, they can be collected into continuous Green's function fields $g_{\mathrm{ij}}(\boldsymbol{v}, \boldsymbol{w}, t)$ and $g_{\mathrm{ij}, \mathrm{k}}(\boldsymbol{v}, \boldsymbol{w}, t)$. When the Green's function fields are appropriately weighted: $\int_{-\infty}^{\infty} \psi_{\mathrm{j}}(\boldsymbol{v}, \boldsymbol{w}) g_{\mathrm{ij}}(\boldsymbol{v}, \boldsymbol{w}, t) \mathrm{d}\|\boldsymbol{v}-\boldsymbol{w}\|$, and $\int_{-\infty}^{\infty} \psi_{\mathrm{jk}}(\boldsymbol{v}, \boldsymbol{w}) g_{\mathrm{ij}, \mathrm{k}}(\boldsymbol{v}, \boldsymbol{w}, t) \mathrm{d}\|\boldsymbol{v}-\boldsymbol{w}\|$, they form volume-tovolume transfer functions which transform an input distributed in a volume $\boldsymbol{v}$ and observed in a volume $\boldsymbol{w}$ to a single output signal. Infinitesimal part $\mathrm{d}\|\boldsymbol{v}-\boldsymbol{w}\|$ represents an infinitesimal change in the distance between points from each of the two volumes.

The purpose of weight functions $\psi_{\mathrm{j}}(\boldsymbol{v}, \boldsymbol{w})$ and $\psi_{\mathrm{jk}}(\boldsymbol{v}, \boldsymbol{w})$ is to incorporate and simplify the spatial source distributions in $v$, the spatial observation sensitivity distributions in $\boldsymbol{w}$, and their relative positions. In this model, the weight functions are calculated by means of geometric probability theory, as described in detail in $[51,53]$.

Returning to the general source from Eq. (3), observed directional displacement $u_{\mathrm{i}}(\boldsymbol{w}, t)$ of a volume $\boldsymbol{w}$ is obtained by convolving the volume-to-volume transfer functions with source impulse magnitudes $J_{\mathrm{j}}, D_{\mathrm{jk}}$ and 
(a)

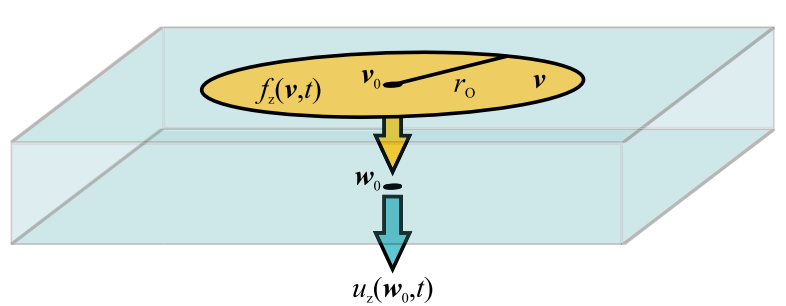

(b)

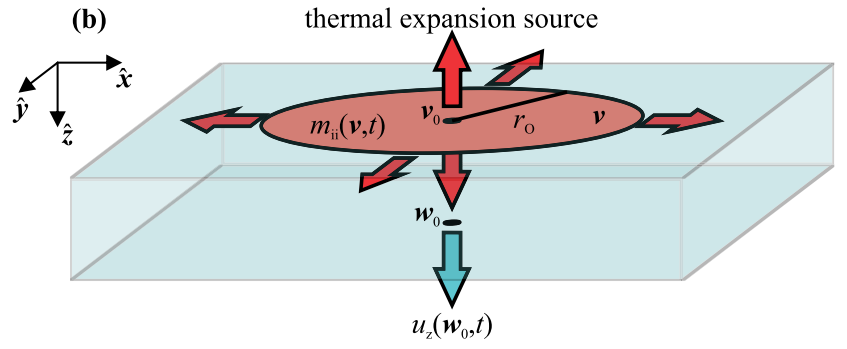

Figure 1: Conceptual presentation of two main laser-stimulated ultrasonic source types that represent two different light-matter interaction mechanisms according to their particular approximations: (a) a pressure source (normal optical force) and (b) a thermal expansion source. From the source volume $\boldsymbol{v}$ centered at a point $\boldsymbol{v}_{0}$, either a distributed force $f_{\mathrm{z}}(\boldsymbol{v}, t)$ or distributed force dipoles $m_{\mathrm{ii}}(\boldsymbol{v}, t)=m_{\mathrm{xx}}(\boldsymbol{v}, t)+m_{\mathrm{yy}}(\boldsymbol{v}, t)+m_{\mathrm{zz}}(\boldsymbol{v}, t)$ generate ultrasonic transients. Ultrasonic waveforms $u_{\mathrm{z}}\left(\boldsymbol{w}_{0}, t\right)$ simulated in this paper are observed at the epicentral point $\boldsymbol{w}_{0}$.

time profiles $\theta(t), \Theta(t)$ :

$$
\begin{gathered}
u_{\mathrm{i}}(\boldsymbol{w}, t)=\sum_{\mathrm{j}} J_{\mathrm{j}} \int_{-\infty}^{\infty} \psi_{\mathrm{j}}(\boldsymbol{v}, \boldsymbol{w}) g_{\mathrm{ij}}(\boldsymbol{v}, \boldsymbol{w}, t) \mathrm{d}\|\boldsymbol{v}-\boldsymbol{w}\| * \theta(t) \hat{\boldsymbol{\imath}}+ \\
\sum_{\mathrm{j}, \mathrm{k}} D_{\mathrm{jk}} \int_{-\infty}^{\infty} \psi_{\mathrm{jk}}(\boldsymbol{v}, \boldsymbol{w}) g_{\mathrm{ij}, \mathrm{k}}(\boldsymbol{v}, \boldsymbol{w}, t) \mathrm{d}\|\boldsymbol{v}-\boldsymbol{w}\| * \Theta(t) \hat{\boldsymbol{\imath}} .
\end{gathered}
$$

Due to commutativity and associativity of convolution in the realm of linearity, the unit step function from the thermal expansion time profile can be moved and convolved with the $\delta$-input Green's functions to form the step-input Green's functions: $h_{\mathrm{ij}, \mathrm{k}}(\boldsymbol{v}, w, t)=$ $g_{\mathrm{ij}, \mathrm{k}}(\boldsymbol{v}, \boldsymbol{w}, t) * H(t)$. Such step-input Green's functions are often more convenient to obtain than $\delta$-input Green's functions.

In the presented model, only the out-of-plane displacements in the $\hat{z}$-direction $u_{\mathrm{z}}(\boldsymbol{w}, t)$ are of interest to us. Compared to radial and azimuthal displacements, such normal displacements are most easily and, therefore, most commonly measured.

When the interaction particularities, discussed previously, are implemented in Eq. (5), together with $\mathrm{i}=\hat{z}$, the out-of-plane displacement calculation is simplified to:

$$
\begin{gathered}
u_{\mathrm{z}}(\boldsymbol{w}, t)=\left(J_{\mathrm{z}} \int_{-\infty}^{\infty} \psi_{\mathrm{z}}(\boldsymbol{v}, \boldsymbol{w}) g_{\mathrm{zz}}(\boldsymbol{v}, \boldsymbol{w}, t) \mathrm{d}\|\boldsymbol{v}-\boldsymbol{w}\|+\right. \\
\left.D \int_{-\infty}^{\infty} \psi_{\mathrm{jj}}(\boldsymbol{v}, \boldsymbol{w}) h_{\mathrm{zj}, \mathrm{j}}(\boldsymbol{v}, \boldsymbol{w}, t) \mathrm{d}\|\boldsymbol{v}-\boldsymbol{w}\|\right) * \theta(t) \hat{z},
\end{gathered}
$$

as $h_{\mathrm{zj}, \mathrm{j}}(\boldsymbol{v}, \boldsymbol{w}, t)=h_{\mathrm{zx}, \mathrm{x}}(\boldsymbol{v}, \boldsymbol{w}, t)+h_{\mathrm{zy}, \mathrm{y}}(\boldsymbol{v}, \boldsymbol{w}, t)+h_{\mathrm{zz}, \mathrm{z}}(\boldsymbol{v}, \boldsymbol{w}, t)$ is used as shorthand.

If, instead of an observation volume $\boldsymbol{w}$, there is an observation point $\boldsymbol{w}_{0}$, the volume-to-volume transfer functions are reduced in complexity to the volume-to-point transfer functions: $\int_{-\infty}^{\infty} \psi_{\mathrm{j}}\left(\boldsymbol{v}, \boldsymbol{w}_{0}\right) g_{\mathrm{ij}}\left(\boldsymbol{v}, \boldsymbol{w}_{0}, t\right) \mathrm{d}\|\boldsymbol{v}-\boldsymbol{w}\|$, and $\int_{-\infty}^{\infty} \psi_{\mathrm{jk}}\left(\boldsymbol{v}, \boldsymbol{w}_{0}\right) g_{\mathrm{ij}, \mathrm{k}}\left(\boldsymbol{v}, \boldsymbol{w}_{0}, t\right) \mathrm{d}\|\boldsymbol{v}-\boldsymbol{w}\|$.

In comparison, a similar arrangement in a commonly used point-to-point approximation degenerates to:

$$
\begin{gathered}
u_{\mathrm{z}}\left(\boldsymbol{w}_{0}, t\right)=\left(J_{\mathrm{z}} g_{\mathrm{zz}}\left(\boldsymbol{v}_{0}, \boldsymbol{w}_{0}, t\right)+\right. \\
\left.D h_{\mathrm{zj}, \mathrm{j}}\left(\boldsymbol{v}_{0}, \boldsymbol{w}_{0}, t\right)\right) * \theta(t) \hat{z}
\end{gathered}
$$

For numerical calculation purposes, it is often convenient to appropriately discretize the forementioned equations.

\subsection{Lateral optical forces}

Light-matter interaction may also produce faint lateral forces which, in turn, produce their own particular transients $[3,4,7,8,9,10,11]$. These forces arise in the same planes as the normal optical forces, but are directed along the interaction surface in the $\hat{x} \hat{y}$-plane and, on the whole, do not transfer any momentum from the incident excitation pulse.

In the spirit of Eq. (4), a source comprising of the lateral optical forces is presented as:

$$
\text { source }_{\tau}\left\{\left(J_{\mathrm{x}} \tilde{\eta}_{\mathrm{x}}(\tilde{\boldsymbol{v}}) \hat{\boldsymbol{x}}+J_{\mathrm{y}} \tilde{\eta}_{\mathrm{y}}(\tilde{\boldsymbol{v}}) \hat{\boldsymbol{y}}\right) \zeta(z) \theta(t),\right.
$$

where $J_{\mathrm{x}}$ and $J_{\mathrm{y}}$ represent the absolute (regardless of orientation) magnitudes of the lateral force impulses distributed within the interaction volume in the directions $\hat{\boldsymbol{x}}$ and $\hat{\boldsymbol{y}}$, respectively. They can be combined to form the absolute (regardless of direction) magnitude of all lateral force impulses distributed within the interaction volume: $J_{\tau}=J_{\mathrm{x}}+J_{\mathrm{y}}$, with their respective shares defined as: $\kappa_{\mathrm{x}} \equiv J_{\mathrm{x}} / J_{\tau}$, and $\kappa_{\mathrm{y}} \equiv J_{\mathrm{y}} / J_{\tau}$.

In the waveform modeling, the directionality of these forces and its combined effect on induced transients must not be discounted. For this reason, the weight functions $\psi_{\mathrm{x}}^{\|}(\boldsymbol{v}, \boldsymbol{w}), \psi_{\mathrm{y}}^{\|}(\boldsymbol{v}, \boldsymbol{w})$ are made to incorporate, 
in addition to the spatial distributions of the source $\tilde{\eta}_{\mathrm{x}}(\tilde{\boldsymbol{v}}) \zeta(z), \tilde{\eta}_{\mathrm{y}}(\tilde{\boldsymbol{v}}) \zeta(z)$, the observational spatial distribution, and their relative positions, the projections of force direction for each point in the source volume $\boldsymbol{v}$ onto a line connecting it with points in the observation volume $\boldsymbol{w}$. Due to symmetry, all of this is lost when observing transients in the epicentral position.

Analogous to Eq. (6), the volume-to-volume model of a displacement waveform induced by the lateral optical forces is written as:

$$
\begin{gathered}
u_{\mathrm{z}}^{\tau}(\boldsymbol{w}, t)=J_{\tau}\left(\kappa_{\mathrm{x}} \int_{-\infty}^{\infty} \psi_{\mathrm{x}}^{\|}(\boldsymbol{v}, \boldsymbol{w}) g_{\mathrm{zx}}(\boldsymbol{v}, \boldsymbol{w}, t) \mathrm{d}\|\boldsymbol{v}-\boldsymbol{w}\|+\right. \\
\left.\kappa_{\mathrm{y}} \int_{-\infty}^{\infty} \psi_{\mathrm{y}}^{\|}(\boldsymbol{v}, \boldsymbol{w}) g_{\mathrm{zy}}(\boldsymbol{v}, \boldsymbol{w}, t) \mathrm{d}\|\boldsymbol{v}-\boldsymbol{w}\|\right) * \theta(t) \hat{z}
\end{gathered}
$$

In comparison, its point-to-point degeneration would only amount to:

$$
\begin{gathered}
u_{\mathrm{z}}^{\tau}\left(\boldsymbol{w}_{0}, t\right)=J_{\tau}\left(\kappa_{\mathrm{x}} g_{\mathrm{zx}}\left(\boldsymbol{v}_{0}, \boldsymbol{w}_{0}, t\right)+\right. \\
\left.\kappa_{\mathrm{y}} g_{\mathrm{zy}}\left(\boldsymbol{v}_{0}, \boldsymbol{w}_{0}, t\right)\right) * \theta(t) \hat{z} .
\end{gathered}
$$

\section{Waveform transition simulations}

In order to present the source enlargement transition as realistically as possible, the waveforms are simulated on a plane-parallel fused silica $\left(\mathrm{SiO}_{2}\right)$ glass plate. Its physical properties are taken as: thickness $h_{0}=12 \mathrm{~mm}$, mass density $\rho=2200 \mathrm{~kg} / \mathrm{m}^{3}$, Young's modulus $Y=$ $7.2 \cdot 10^{10} \mathrm{~Pa}$, Poisson's ratio $\mu=0.17$, specific heat $C=$ $700 \mathrm{~J} /(\mathrm{kg} \mathrm{K})$, and linear thermal expansion coefficient $\alpha=5.6 \cdot 10^{-7} / \mathrm{K}$, making the propagation velocity of primary $(\mathrm{P})$ waves $c_{\mathrm{P}}=5931 \mathrm{~m} / \mathrm{s}$ and of secondary $(\mathrm{S})$ waves $c_{\mathrm{S}}=3740 \mathrm{~m} / \mathrm{s}$.

The ultrasonic source is modeled as if it was stimulated by a laser pulse impacting on a plate surface at $z=0$ with a uniform (top-hat) areal distribution $\tilde{\eta}(\tilde{\boldsymbol{v}})$ over a circular surface area $\tilde{\boldsymbol{v}}$ with a fixed central point $\boldsymbol{v}_{0}$ and a radius $r_{\mathrm{O}}$ that is expanded from (nearly) a point $\left(r_{O} \rightarrow 0\right)$ to a quasi-limitless extent $\left(r_{\mathrm{O}} \rightarrow \infty\right)$ while retaining a constant fluence of $q=0.2 \mathrm{~J} / \mathrm{mm}^{2}$. Keeping the fluence at constant levels allows for a more illuminating comparison of the successive waveforms with the understanding that an increase in source radius directly increases the delivered energy as well. Its normalized time profile has the shape of a double sigmoidal (asymmetric bell-like) function with a full width at half maximum of $t_{\Leftrightarrow}=60 \mathrm{~ns}$. Since the numerical calculations require discretized equations, the radial resolution of the source enlargement is $\mathrm{d} r=18 \mu \mathrm{m}$.

The observation point $\boldsymbol{w}_{0}$, rather than a volume, is always set in the epicentral position on the opposite surface directly beneath the central point of the source, as shown in Fig. 1. The waveforms are modeled within the observation time frame of $t_{\Omega}=12.15 \mu \mathrm{s}$ with temporal resolution of $\mathrm{d} t=3 \mathrm{~ns}$. As described previously, they are constructed out of discretized statistically weighted combinations of Green's functions [51, 53] whose calculation by means of generalized ray theory was derived and programed by Hsu [31]. For this kind of epicentral arrangement with a circular uniformly distributed surface source, the required weight functions are quite straightforward: $\psi\left(\boldsymbol{v}, \boldsymbol{w}_{0}\right)=2 r / r_{\mathrm{O}}^{2}$, where $r$ is the internal radial parameter and runs from 0 to $r_{\mathrm{O}}$.

The term 'quasi-limitless extent' is used to articulate that the source is sufficiently large so that the fastest ultrasonic transients originating from its edge do not reach the observation point in the observable time frame. With the parameters used here, the quasi-limitless source has a radius $r_{t>t_{\Omega}}>71 \mathrm{~mm}$.

To appreciate their distinctions, the ultrasonic waveforms induced by each of the induction mechanisms are modeled and presented separately.

For the pressure-induced ultrasound, the laser pulse is reflected in its entirety from the impact surface at $z=0$. Its reflectivity is thus set to $R=1$ while the source depth distribution is approximated as: $\zeta(z)=\delta(z)$.

For the thermal-expansion-induced ultrasound, however, the laser pulse is fully absorbed in a thin layer set beneath the impact surface at the average depth of light penetration of $z=\delta h=10 \mu \mathrm{m}$. For this, the absorptivity of the layer is set to $A=1$ while the source depth distribution is approximated as: $Z(z)=\delta(z-\delta h)$. To bury an expansion source inside the material means to account for its isotropical expansion better than have it directly on the surface where vertical components vanish in this modeling method [13, 14, 38, 39, 41, 43, 44].

Reducing each of the source depth distributions into infinitesimally thin planes of interaction greatly simplifies the modeling as, in effect, it reduces volumetric sources into areal sources. Experimentally, on a glass plate, a mirror-like surface can be achieved with a highreflectivity (HR) surface coating while, on another plate, a thin highly absorptive volume can be made with a high-absorptivity (HA) metal coating. If such coatings are sufficiently thin and have similar elastic properties as the main substrate, they have no direct effect on wave propagation.

The pressure-induced displacement waveform transition simulation during the source enlargement is pre- 

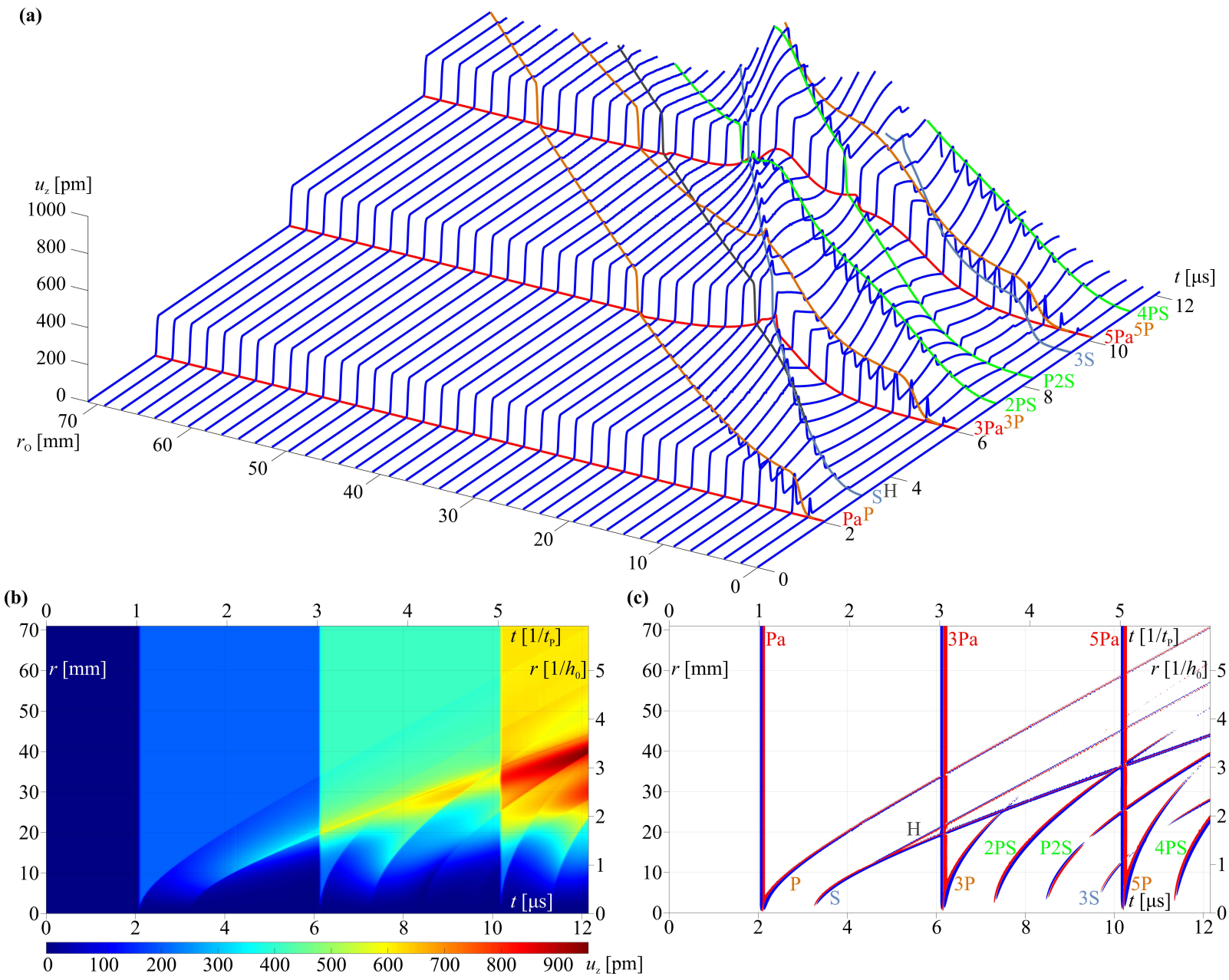

Figure 2: Simulation of pressure-induced surface out-of-plane displacement waveforms $u_{\mathrm{z}}$ in the epicentral position in a large glass plate as functions of time $t$ and with regard to the source radius $r_{\mathrm{O}}$ in a transition from a point to a quasi-limitless extent. They are presented as (a) a three-dimensional line plot with explicitly marked wave arrivals, (b) its top-down view as a continuous shaded surface plot, and (c) a wave arrival plot obtained as a Laplacian of the latter.

sented in Fig. 2 and its thermal-expansion-induced counterpart is presented in Fig. 3. Figures 2a and 3a show three-dimensional waveform line plots at regular source radius enlargement intervals with identified wave types and their arrival times. These are discussed further on. A top-down view of these plots with a color-coded surface displacements and a continuous source radius enlargements are presented in Figs. $2 \mathrm{~b}$ and $3 \mathrm{~b}$. A Laplacian of these surface plots gives the curvature of the waveforms and, with appropriately chosen color coding, can be made to show the wave arrival times directly from the simulated waveforms. In Figs. $2 \mathrm{c}$ and $3 \mathrm{c}$, such wave arrival times are visualized with red representing a negative peak curvature and blue representing a positive peak curvature. Each of the waveform transition sim- ulations is also animated in the supplementary videos on-line.

Selected waveforms for distinct source sizes produced by each induction mechanism separately are presented in Fig. 4. A point source, an areal source with $r_{\mathrm{O}}=24 \mathrm{~mm}$, and a quasi-limitless source waveforms are compared with each-other and with independent models from the literature: a doubled analytical areal source model for a half-space $[20,40]$, and a onedimensional analytical homogeneous source model [2].

\section{Discussion}

Combining pressure and thermal expansion induction mechanisms into a single ultrasonic source provides a 

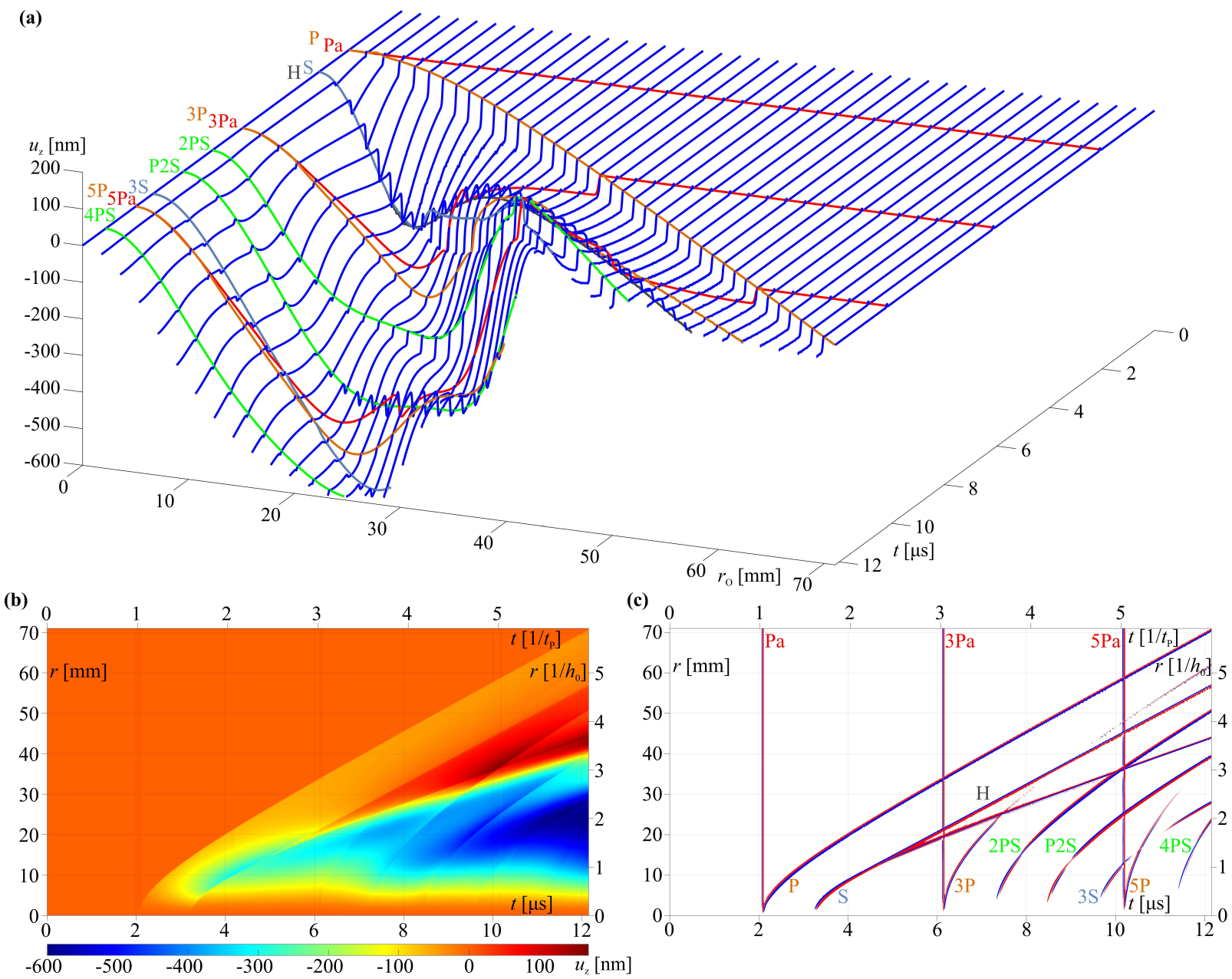

Figure 3: Simulation of thermal-expansion-induced surface out-of-plane displacement waveforms $u_{\mathrm{z}}$ in the epicentral position in a large glass plate as functions of time $t$ and with regard to the source radius $r_{\mathrm{O}}$ in a transition from a point to a quasi-limitless extent. They are presented as (a) a three-dimensional line plot with explicitly marked wave arrivals, (b) its top-down view as a continuous shaded surface plot, and (c) a wave arrival plot obtained as a Laplacian of the latter.

conceptual insight on how realistic ultrasonic transients in elastic materials are generated by laser illumination. While treating them separately reveals their individual intricacies, it is their combination that genuinely describes the light-matter interaction that takes place below the ablation threshold.

The choice of statistically enhanced Green's function formalism as a method for this kind of modeling, as opposed to analytical solutions or a finite element method, was guided by several aspects in their comparison. Fully analytical solutions are most often only achievable for the simplest of geometries and very rarely, if at all, for complex macroscopic sources in plate substrates with several reverberating transients traveling through them. They do, however, allow for a convenient play with material parameters. While finite element and finite difference methods have the ability to account for almost any complexity, that power may quickly overwhelm the computing process which has to be restarted each time a new variation is desired. In this, the chosen method tends to be much faster, particularly when introducing limited arrangement variations to a known Green's function field or a discretized set of Green's functions where only the weight function is, relatively quickly, recalculated. Although a simplification, it is the geometric probability of the statistically streamlined Huygens' principle by which the arbitrary spatial and even directional distributions of complex sources together with the spatial observation sensitivity distribution are incorporated in the weight func- 

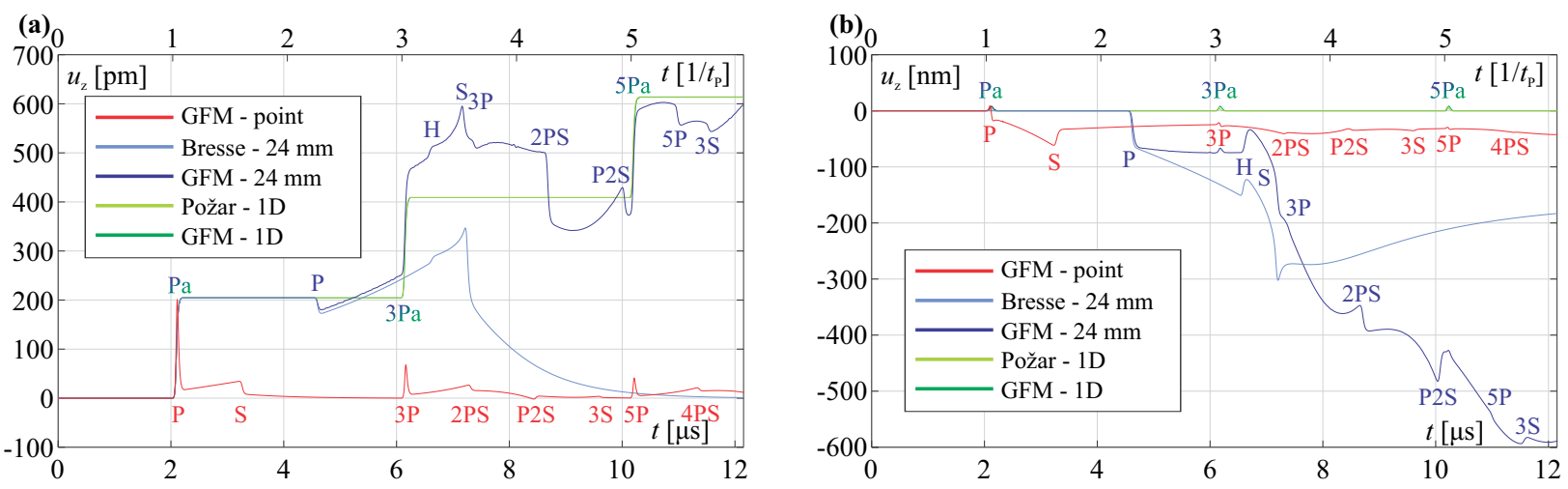

Figure 4: Comparison between representative ultrasonic (a) pressure-induced and (b) thermal-expansion-induced out-of-plane surface displacement waveforms $u_{\mathrm{z}}$ with identified transient types for three distinct source sizes. A point source (point), an areal source with $r_{\mathrm{O}}=2 h_{0}=24 \mathrm{~mm}$, and a quasi-limitless source (1D) waveforms are modeled using statistically enhanced Green's function method (GFM) and compared with doubled analytical areal source model for a half-space (Bresse) [20, 40] and one-dimensional analytical homogeneous source model (Požar) [2].

tions that guarantees that these complexities are not lost in the modeling. When calculating Green's functions using the generalized ray theory methods, the ability to exclude certain wave types may present itself as another advantage over the two numerical methods which provide only complete solutions.

The chosen method is only possible because the formulations are assumed to inhabit the cross section of linear electrodynamics and linear elastodynamics which enables many otherwise complex mathematical operations to be substituted by a series of simpler ones. Operating in such a realm provides an optimal compromise between the complexity of calculations and the complexity of results, thus, achieving a comprehensive simplicity for the purposes of the waveform modeling.

The simulations of the epicentral ultrasonic waveforms as induced by the pressure and thermal expansion sources in transition from a point to a quasi-limitless extent from Figs. 2 and 3 show expected characteristics, as inferred from the various sources in the literature $[12,13,14,15,16,17,18,19,20,21,22,23,24,25,26$, $27,28,29,30,31,32,33,34,35,36,37,38,39,40,41$, $42,43,44]$. In fact, they show much more as the surface source enlargement seamlessly connects the two limiting extremes. Although the waveforms departing from a point limit change gradually as they transition toward the one-dimensional limit, the extremes are so dissimilar that their connection seems inexplicable without explicitly showing such an evolution.

Comparing the surface displacement waveforms as induced by the two generation mechanisms illuminates fundamental distinctions and similarities between them. The amplitude ranges in the simulated timespan for the specific material under those conditions diverge by approximately three orders of magnitude in favor of thermal-expansion-induced waveforms over the pressure-induced ones. It is the complete energy absorption, as simulated here, that contributes far more to the material displacement than any momentumtransferring pulse reflection strike. Simultaneously, the pressure-induced surface displacements are exclusively positive (out of plane) while those induced by thermal expansion are predominantly negative (into the bulk). Most apparent similarity between them is the corresponding presence of all wave types and their arrival times throughout the source enlargement as identified in the figures and specifically visualized in Figs. $2 \mathrm{c}$ and 3c.

The waveforms are superpositions of several individual transients which can be identified as primary (longitudinal) P-waves (P, 3P, 5P...), secondary (transversal) S-waves (S, 3S...), combinations of the two due to mode conversion (2PS, P2S, 4PS...), and a head wave (H). The numbers in the wave type nomenclature indicate the number of passes of that specific wave type through the bulk of the medium which is one more than the number of its reflections from the surfaces.

Another important distinction can be found between those transients originating from the source interior $(\mathrm{Pa}$, $3 \mathrm{~Pa}, 5 \mathrm{~Pa} . .$.$) and those originating from the source edge$ (the rest). This becomes apparent when examining the transition of the waveforms as only the edge-originating waves can be observed in the point limit, only the interior-originating waves can be observed in the onedimensional limit and all of the waves can be seen between the limits. A quick study of wave arrivals yields 
wave-specific time of arrival equations:

$$
\begin{aligned}
t_{n \mathrm{~Pa}} & =n h_{0} / c_{\mathrm{P}}, & n=1,3,5 \ldots, \\
t_{n \mathrm{P}} & =\sqrt{r_{\mathrm{O}}^{2}+\left(n h_{0}\right)^{2}} / c_{\mathrm{P}}, & n=1,3,5 \ldots, \\
t_{n \mathrm{~S}} & =\sqrt{r_{\mathrm{O}}^{2}+\left(n h_{0}\right)^{2}} / c_{\mathrm{S}}, & n=1,3 \ldots, \\
t_{\mathrm{H}} & =r_{\mathrm{O}} / c_{\mathrm{P}}+h_{0} \sqrt{c_{\mathrm{S}}^{-2}-c_{\mathrm{P}}^{-2}} . &
\end{aligned}
$$

They confirm that arrival times of interior-originating transients $\left(t_{n \mathrm{~Pa}}\right)$ bear no dependence on the source radius $r_{\mathrm{O}}$, where its edge is, while those of edge-originating transients do. Equations similar to the middle two, though rather more complex, may be found to hold for mode-converted transients as well. There is a curiosity at $r_{\mathrm{O}} \gg n h_{0}$ where: $t_{n \mathrm{P}} \approx r_{\mathrm{O}} / c_{\mathrm{P}}$ and $t_{n \mathrm{~S}} \approx r_{\mathrm{O}} / c_{\mathrm{S}}$, which shows that all pure primary and secondary waves (not mode-converted) have their own asymptotic functions for large enough $t$.

The exclusive outward surface displacement of the pressure-induced waves and its persistence at larger times indicate that the $\hat{z}$-directed momentum has indeed been transferred from the incident laser pulse to the substrate. In particular, the step-like profiles at distinct times $t_{n \mathrm{~Pa}}$ for larger source sizes demonstrate a forward motion of the entire material-propelled only by the laser illumination.

In contrast, the short-lived predominantly inward surface displacement in thermal-expansion-induced waveforms is a consequence of the edge waves with the first P-wave acting as a delimiter between them and the mostly level parts of waveforms as the source grows larger. Barely noticeable outward precursors that remain at distinct times $t_{n \mathrm{~Pa}}$ are produced by the normal component of the force dipoles. While all the neighboring components in $\hat{\boldsymbol{x}} \hat{\boldsymbol{y}}$-plane annihilate each-other, the $+\hat{z}$-directed dipole component transients become superimposed with the $-\hat{z}$-directed dipole component transients upon their reflection from the incident surface with enough of a shift between them to produce the small outward precursors [13, 14, 38, 39, 41, 43, 44]. The nearly flat waveform profiles for larger source sizes with reverting precursors confirm that, on the whole, no $\hat{z}$-directed momentum has been transferred from the incident laser pulse to the bulk of the material.

As another point of interest, some of the waves, the P2S-wave for example, exhibit a polarity inversion during the source enlargement which is best observed in Figs. $2 c$ and $3 c$ where the curvature colors exchange their relative positions.

Representative waveforms from each of the limiting sources and for the source with radius $r_{\mathrm{O}}=24 \mathrm{~mm}$ are compared with each-other and with the corresponding independent waveforms obtained from the literature $[2,20,40]$ in Fig. 4. In addition to vividly showing seemingly unconnected profiles of the limiting waveforms, it also shows a good match between the corresponding waveforms calculated by different modeling methods. It has to be noted that Bresse's analytical models [20, 40] are valid only for a half-space. They do not include any of the reflected transients nor do they include the precursors in the thermal-expansion-induced waveforms. They also had to be doubled to account for the amplitude doubling during the wave reflection from the plate surface.

Faithful simulations of waveforms induced by the lateral optical forces require the knowledge of their spatial and directional distributions which, in turn, require deeper understanding of light-matter interaction mechanisms, more specifically, the final resolution of the socalled Abraham-Minkowski controversy. Such measurements are conceivable but still necessitate yet further evolution in measuring techniques.

\section{Conclusions}

We presented a combined derivation of pressureinduced and thermal-expansion-induced ultrasonic waves in elastic media from a common laser-stimulated source. Next to the normal optical forces, the lateral optical forces were considered as well. Using the derived model, for the particular case of a solid glass plate and a stimulating laser pulse, the transient waveforms were calculated in the epicentral position. They were shown for surface sources in transition from a point to a quasi-limitless extent induced by each of the two main induction mechanisms as they might be produced by an incident laser pulse. Behaviour of individual wave types, as influenced by the source size and the induction mechanisms, was discussed.

\section{Acknowledgements}

The authors acknowledge the financial support from the Slovenian Research Agency (research core funding No. P2-0392).

\section{References}

[1] T. Požar, J. Možina, Measurement of elastic waves induced by the reflection of light, Phys. Rev. Lett. 111 (2013) 185501, http://dx.doi.org/10.1103/PhysRevLett.111.185501. 
[2] T. Požar, A. Babnik, J. Možina, From laser ultrasonics to optical manipulation, Opt. Express 23 (2015) 7978-7990, http://dx.doi.org/10.1364/OE.23.007978.

[3] B.A. Kemp, Resolution of the Abraham-Minkowski debate: Implications for the electromagnetic wave theory of light in matter, J. Appl. Phys. 109 (2011) 111101, http://dx.doi.org/10.1063/1.3582151.

[4] M. Bethune-Waddell, K.J. Chau, Simulations of radiation pressure experiments narrow down the energy and momentum of light in matter, Rep. Prog. Phys. 78 (2015) 122401, http://dx.doi.org/10.1088/0034-4885/78/12/122401.

[5] N.G.C. Astrath, G.V.B. Lukasievicz, L.C. Malacarne, S.E. Bialkowski, Surface deformation effects induced by radiation pressure and electrostriction forces in dielectric solids, Appl. Phys. Lett. 102 (2013) 231903, http://dx.doi.org/10.1063/1.4809924.

[6] O.A. Capeloto, V.S. Zanuto, L.C. Malacarne, M.L. Baesso, G.V.B. Lukasievicz, S.E. Bialkowski, N.G.C. Astrath, Quantitative assessment of radiation force effect at the dielectric air-liquid interface, Scientific Reports 6 (2016) 20515, http://dx.doi.org/10.1038/srep20515.

[7] M. Mansuripur, Radiation pressure and the linear momentum of the electromagnetic field, Opt. Express 12 (2004) 5375-5401, https://doi.org/10.1364/OPEX.12.005375.

[8] A.R. Zakharian, M. Mansuripur, J.V. Moloney, Radiation pressure and the distribution of electromagnetic force in dielectric media, Opt. Express 13 (2005) 2321-2336, https://doi.org/10.1364/OPEX.13.002321.

[9] M. Mansuripur, A.R. Zakharian, E.M. Wright, Electromagneticforce distribution inside matter, Phys. Rev. A 88 (2013) 023826, https://doi.org/10.1103/PhysRevA.88.023826.

[10] M. Mansuripur, Resolution of the Abraham-Minkowski controversy, Opt. Commun. 283 (2010) 1997?2005, http://dx.doi.org/10.1016/j.optcom.2010.01.010.

[11] M. Mansuripur, A.R. Zakharian, Whence the Minkowski momentum?, Opt. Commun. 283 (2010) 3557-3563, http://dx.doi.org/10.1016/j.optcom.2010.04.059.

[12] J.D. Achenbach, Wave propagation in elastic solids, NorthHolland publishing company, Amsterdam, 1973.

[13] D. Royer, E. Dieulesaint, Elastic waves in solids II: generation, acousto-optic interaction, applications, Springer-Verlag, Berlin, 2000.

[14] C.B. Scruby, L.E. Drain, Laser ultrasonics: techniques and applications, Adam Hilger, Bristol, 1990.

[15] V.E. Gusev, A.A. Karabutov, Laser optoacoustics, American Institute of Physics, New York, 1993.

[16] C.U. Grosse, M. Ohtsu, Acoustic emission testing, SpringerVerlag, Berlin, 2008.

[17] E. Kausel, Fundamental Solutions in Elastodynamics: A Compendium, Cambridge University Press, Cambridge, 2011

[18] S.J. Davies, C. Edwards, G.S. Taylor, S.B. Palmer, LaserGenerated Ultrasound: Its Properties, Mechanisms and Multifarious Applications, J. Phys. D-Appl. Phys. 26 (1993) 329-348, http://dx.doi.org/10.1088/0022-3727/26/3/001.

[19] T. Požar, J. Možina, 1D problems of radiation pressure on elastic solids, Proc. SPIE 9548 (2015) 95480N, http://dx.doi.org/10.1117/12.2191696.

[20] L.F. Bresse, D.A. Hutchins, Transient generation of elastic waves in solids by a disk-shaped normal force source, J. Acoust. Soc. Am. 86 (1989) 810-817, http://dx.doi.org/10.1121/1.398204.

[21] A. McNab, A. Cochran, M.A. Campbell, The calculation of acoustic fields in solids for transient normal surface force sources of arbitrary geometry and apodization, J. Acoust. Soc. Am. 87 (1990) 1455-1465, http://dx.doi.org/10.1121/1.399442.
[22] A. Lhémery, A model for the transient ultrasonic field radiated by an arbitrary loading in a solid, J. Acoust. Soc. Am. 96 (1994) 3776-3786, http://dx.doi.org/10.1121/1.410566.

[23] E. Bécache, A.P. Kiselev, Non-stationary elastic wavefields from an apodized normal transducer. Near-field asymptotics and numerics, Acta. Acust. United Ac. 91 (2005) 822-830, https://hal-ensta.archives-ouvertes.fr/hal-00982677.

[24] J.P. Weight, A model for the propagation of short pulses of ultrasound in a solid, J. Acoust. Soc. Am. 81 (1987) 815-826, http://dx.doi.org/10.1121/1.394560.

[25] H. Douglas Mair, L. Bresse, D.A. Hutchins, Diffraction effects of planar transducers using a numerical expression for edge waves, J. Acoust. Soc. Am. 84 (1988) 1517-1525, http://dx.doi.org/10.1121/1.396598.

[26] A.G. Every, K.Y. Kim, Time domain dynamic response functions of elastically anisotropic solids, J. Acoust. Soc. Am. 95 (1994) 2505-2516, http://dx.doi.org/10.1121/1.409860.

[27] C. Rouge, A. Lhémery, D. Ségur, Transformation of body force localized near the surface of a halfspace into equivalent surface stresses, J. Acoust. Soc. Am. 134 (2013) 2639-2646, http://dx.doi.org/10.1121/1.4816489.

[28] E. Kausel, Lamb's problem at its simplest, Proc. R. Soc. A 469 (2013) 20120462, http://dx.doi.org/10.1098/rspa.2012.0462.

[29] A.N. Ceranoglu, Y.H. Pao, Propagation of Elastic Pulses and Acoustic Emission in a Plate-Part 1: Theory, J. Appl. Mech. 48 (1981) 125-132, http://dx.doi.org/10.1115/1.3157554.

[30] A.N. Ceranoglu, Y.H. Pao, Propagation of Elastic Pulses and Acoustic Emission in a Plate-Part 3: General Responses, J. Appl. Mech. 48 (1981) 139-147, http://dx.doi.org/10.1115/1.3157556.

[31] N.N. Hsu, Dynamic Green's functions of an infinite plate - a computer program, Technical Report No. NBSIR 85-3234, National Bureau of Standards, Washington, 1985.

[32] J.D. Aussel, A. Le Brun, J.C. Baboux, Generating acoustic waves by laser: theoretical and experimental study of the emission source, Ultrasonics 26 (1988) 245-255, http://dx.doi.org/10.1016/0041-624X(88)90013-3.

[33] A.N. Ceranoglu, Y.H. Pao, Propagation of Elastic Pulses and Acoustic Emission in a Plate-Part 2: Epicentral Responses, J. Appl. Mech. 48 (1981) 133-138, http://dx.doi.org/10.1115/1.3157555.

[34] S. Fassbender, B. Hoffmann, W. Arnold, Efficient Generation of Acoustic Pressure Waves by Short Laser Pulses, Mat. Sci. Eng. A-Struct. 122 (1989) 37-41, http://dx.doi.org/10.1016/09215093(89)90768-5.

[35] R.J. Dewhurst, D.A. Hutchins, S.B. Palmer, C.B. Scruby, Quantitative measurements of laser-generated acoustic waveforms, J. Appl. Phys. 53 (1982) 4064-4071, http://dx.doi.org/10.1063/1.331270.

[36] C.B. Scruby, R.J. Dewhurst, D.A. Hutchins, S.B. Palmer, Quantitative studies of thermally generated elastic waves in laser-irradiated metals, J. Appl. Phys. 51 (1980) 6210-6216, http://dx.doi.org/10.1063/1.327601.

[37] L.R.F. Rose, Point-source representation for laser-generated ultrasound, J. Acoust. Soc. Am. 75 (1984) 723-732, http://dx.doi.org/10.1121/1.390583.

[38] P.A. Doyle, On epicentral waveforms for laser-generated ultrasound, J. Phys. D: Appl. Phys. 19 (1986) 1613-1623, http://dx.doi.org/10.1088/0022-3727/19/9/007.

[39] K.L. Telschow, R.J. Conant, Optical and thermal parameter effects on laser-generated ultrasound, J. Acoust. Soc. Am. 88 (1990) 1494-1502, http://dx.doi.org/10.1121/1.400306.

[40] L.F. Bresse, D.A. Hutchins, Transient generation by a wide thermoelastic source at a solid surface, J. Appl. Phys. 65 (1989) 1441-1446, http://dx.doi.org/10.1063/1.342956. 
[41] A.G. Every, Z.N. Utegulov, I.A. Veres, Laser thermoelastic generation in metals above the melt threshold, J. Appl. Phys. 114 (2013) 203508, http://dx.doi.org/10.1063/1.4832483.

[42] S. Dixon, T. Harrison, Y. Fan, P.A. Petcher, Thermoelastic laser generated ultrasound using a ring source, J. Phys. D: Appl. Phys. 45 (2012) 175103, http://dx.doi.org/10.1088/00223727/45/17/175103.

[43] F.A. McDonald, On the precursor in laser-generated ultrasound waveforms in metals, Appl. Phys. Lett. 56 (1990) 230-232, http://dx.doi.org/10.1063/1.102839.

[44] F. Enguehard, L. Bertrand, Temporal deconvolution of laser-generated longitudinal acoustic waves for optical characterization and precise longitudinal acoustic velocity evaluation, J. Acoust. Soc. Am. 103 (1998) 771-784, http://dx.doi.org/10.1121/1.421241.

[45] K. Aki, P.G. Richards, Quantitative Seismology, University Science Books, Sausalito, 2002.

[46] S. Stein, M. Wysession, An introduction to seismology earthquakes and earth structure, Blackwell Publishing, Malden, 2003.

[47] J. Pujol, Elastic wave propagation and generation in seismology, Cambridge University Press, Cambridge, 2003.

[48] G.C. McLaskey, D.A. Lockner, B.D. Kilgore, N.M. Beeler, A robust calibration technique for acoustic emission systems based on momentum transfer from a ball drop, B. Seismol. Soc. Am. 105 (2015) 257-271, http://dx.doi.org/10.1785/0120140170.

[49] B.W. Stump, L.R. Johnson, The determination of source properties by the linear inversion of seismograms, B. Seismol. Soc. Am. 67 (1977) 1489-1502, http://www.bssaonline.org/content/67/6/1489.abstract.

[50] L. Gualtieri, G. Ekström, Seismic Reconstruction of the 2012 Palisades Rockfall Using the Analytical Solution to Lamb's Problem, B. Seismol. Soc. Am. 107 (2017), http://dx.doi.org/10.1785/0120160238

[51] J. Laloš, A. Babnik, J. Možina, T. Požar, Incorporation of a spatial source distribution and a spatial sensor sensitivity in a laser ultrasound propagation model using a streamlined Huygens' principle, Ultrasonics 66 (2016) 34-42, http://dx.doi.org/10.1016/j.ultras.2015.12.002.

[52] J. Laloš, T. Požar, J. Možina, High-frequency calibration of piezoelectric displacement sensors using elastic waves induced by light pressure, Stroj. Vestn.-J. Mech. E. 61 (2015) 533-542, http://dx.doi.org/10.5545/sv-jme.2015.2731.

[53] J. Laloš, T. Požar, J. Možina, Transition from 1D to 2D laserinduced ultrasonic wave propagation in an extended plate, Int. J. Thermophys. 37 (2016) 52, http://dx.doi.org/10.1007/s10765016-2056-y.

[54] A.M. Mathai, An introduction to geometrical probability: Distributional aspects with applications, Gordon and Breach, Newark, 1999.

[55] K. Oldham, J. Myland, J. Spanier, An Atlas of Functions, Springer-Verlag, New York, 2009, http://dx.doi.org/10.1007/978-0-387-48807-3. 